\title{
Variable observation window length blind equalization detector for underwater acoustic communication
}

\author{
Zhiyong Liu* ${ }^{*}$, Fan Bai and Zhoumei Tan
}

$\bar{*}$ *Correspondence: Izyhit@hit.edu.cn School of Information Science and Engineering, Harbin Institute of Technology, West Wenhua Road, Weihai 264209, China

\begin{abstract}
Aiming at the influence of underwater acoustic channel (UAC) profile difference on the performance of underwater acoustic communication, a variable observation window length blind equalization detector (VOWL-BED) is proposed in this paper. Compared with the existing methods, the observation window length (OWL) of the proposed VOWL-BED can be dynamically regulated on the basis of a given UAC profile and finally converge to the optimum OWL. We have verified the feasibility of the OWL regulation. The simulation results also demonstrate that the VOWL-BED can achieve better performance than the conventional invariable OWL blind equalization detector (IOWL-BED).
\end{abstract}

Keywords: Underwater acoustic communication, Variable observation window length, Blind equalization detector, Intersymbol interference

\section{Introduction}

Underwater acoustic channel (UAC) is one of the most complex wireless channels. In such channels, intersymbol interference (ISI) is caused by the overlap of symbols, due to the multipath signal propagation [1-3]. Severe ISI is one of the important factors degrading the system performance of underwater acoustic communication.

To achieve reliable communication over the underwater acoustic channels, many equalization techniques have been developed to mitigate the ISI. Most of the existing equalization techniques work in the traditional non-blind mode [4-7], the training process is indispensable, and the tap coefficient vector is obtained based on the training sequences. However, the bandwidth of UAC is limited [1,3]. The actual utilization for limited frequency band is enormously reduced by the use of training sequences. To use the restricted bandwidth of UAC more efficiently, some blind equalization approaches are applied to underwater acoustic communication [8-31]. In the blind equalization, the updating of tap coefficient vector takes advantage of the priori knowledge of transmitted signal statistics rather than the training sequences. Constant modulus algorithm (CMA) and multimodulus algorithm (MMA) are the two most approved blind equalization algorithms. In [8-24], the CMA and the improved CMA are applied to

(c) The Author(s). 2020 Open Access This article is licensed under a Creative Commons Attribution 4.0 International License, which permits use, sharing, adaptation, distribution and reproduction in any medium or format, as long as you give appropriate credit to the original author(s) and the source, provide a link to the Creative Commons licence, and indicate if changes were made. The images or other third party material in this article are included in the article's Creative Commons licence, unless indicated otherwise in a credit line to the material. If material is not included in the article's Creative Commons licence and your intended use is not permitted by statutory regulation or exceeds the permitted use, you will need to obtain permission directly from the copyright holder. To view a copy of this licence, visit http://creativecommons.org/licenses/by/4.0/. 
underwater acoustic communication. To implement phase recovery in the constellation, the rotator needs to be added to the output of the CMA blind equalizer, and thus, the complexity of the receiver is increased. The MMA can simultaneously complete the blind equalization and the phase recovery, eliminating the requirement for the rotator. For underwater acoustic communication system with QAM modulation, the equalization performance can be improved by using the MMA [25-28]. In addition, at the cost of increasing complexity, the structure of blind equalization in conjunction with a multichannel combiner has been proven to be effective for improving system performance [29-31].

The equalization performance based on minimum mean square error (MMSE) standard has been comprehensively analyzed, the results show that the MMSE obtained by the equalizer is a monotonically non-increasing function of the observation window length (OWL) [32, 33], and the OWL would severely influence the performance of equalizer. To achieve optimal equalization performance, one of the main challenges is the selection of the OWL. Several variable OWL schemes for non-blind equalization algorithm are proposed for terrestrial wireless communication environment [34-39]; in these schemes, the channel is assumed to be stationary, and the observation period is infinitely long. However, in underwater scenarios, the channel exhibits obvious non-stationarity. The number of stationary observations, which can be obtained within a certain time interval, is restricted by the variability of UAC with time changing [40]. The finite number of stationary observations used to adaptively adjust the OWL in turn restricts the adaptive and effective selection of OWL, which makes the OWL adaptation difficult to accomplish under the underwater acoustic environment. Blind equalization provides a possible solution to the problem; because the training sequence is not required, the algorithm is implemented with the priori knowledge of transmitted signal statistic. However, in all previous formulations of the blind equalization, the OWL is assumed to be fixed [8-31]. Moreover, in different time and different underwater environment, the channel profile is different, and the optimal OWL for blind equalization is related with the specific channel profile. Therefore, in practical underwater acoustic communication, the optimal OWL is difficult to obtain in advance. The performance of blind equalization is seriously affected by the OWL. If the OWL is too long, it would increase the computational complexity of blind equalizer; on the contrary, in case the OWL is too short, the required blind equalization performance may not be obtained. Hence, to accomplish better blind equalization performance, the blind equalizer needs to have the ability to dynamically regulate and search the optimum OWL in the light of the given UAC profile. As far as we know, the investigations on variable OWL blind equalization detector (VOWL-BED) for underwater acoustic communication are still lacking.

In this paper, a VOWL-BED for underwater acoustic communication is proposed. The detector can adaptively regulate the OWL on the basis of the given UAC profile. Moreover, the proposed blind equalization detector does not need the training sequences and thus can improve the transmission efficiency. As a result, for the actual underwater acoustic environment, the proposed VOWL-BED is more feasible for the realization of the underwater acoustic communication systems. Overall, the main contributions of the paper can be summarized as follows: 
- We propose an underwater acoustic communication system based on blind equalization detector. Because no training sequence is needed by the BED, the transmission efficiency of system is improved.

- To achieve better performance, a variable observation window length blind equalization detector base on accumulated squared error (ASE) is also proposed. The OWL of the detector can be adjusted adaptively according to the profile of the specific channel.

- The experiments of this paper are carried out based on MATLAB platform. To better simulate the real underwater acoustic scenarios, the Bellhop model is used to produce the underwater acoustic channel. We conduct designed simulation to verify the feasibility of adjusting OWL for BED. We also verify that the proposed VOWL-BED can achieve better performance than traditional invariable OWL blind equalization detector (IOWL-BED).

The rest of this paper is organized as follows. Section 2 presents the considered system model and the existing IOWL-BED. The proposed VOWL-BED is introduced in Section 3. The simulation results and discussion are given in Section 4. Finally, conclusions are drawn in Section 5.

\section{System model and conventional method}

\subsection{System model}

We consider a point-to-point underwater acoustic communication system, in which the quadrature amplitude modulation (QAM) is adopted. For the transmitter, the transmitted signal can be given by:

$$
x(t)=\sum_{i=1}^{N_{d}} d(i) p\left(t-i T_{s}\right)
$$

where $N_{d}$ is the number of symbols per data packet, $T_{s}$ represents the symbol period, $d(j)$ denotes the equiprobable and statistical independent 4-QAM data stream, and $p(t)$ represents the pulse waveform of the transmitted symbol. After the transmitted signal $x(t)$ is transmitted via the UAC, the received signal can be written as:

$$
u(t)=x(t) * h_{I R}(t)+n(t)
$$

where * expresses the convolution operation, $n(t)$ denotes the additive white Gaussian noise (AWGN), and $h_{I R}(t)$ is the impulse response of UAC, and can be obtained by the Bellhop model $[41,42]$.

The received complex baseband signal $u(t)$ is firstly sampled with the symbol inter$\mathrm{val}$, and then, the sampled signals $u(k)$ are used as the input of the BED. For the symbol interval sampled signals, the BED correspondingly adopts the structure of symbol spaced linear filter. In the blind equalization algorithm, the signal vector composed of $u(k)$ is given by:

$$
\boldsymbol{v}(k)=[u(k-1), u(k-2), \cdots, u(k-M)]
$$

where $M$ is the OWL of BED. The homologous tap coefficient vector can be expressed as:

$$
\boldsymbol{w}(k)=\left[w_{k 1}, w_{k 2}, \cdots, w_{k M}\right]
$$


where $\boldsymbol{w}(k)$ is initialized with $\boldsymbol{w}=[\overbrace{0, \cdots, 0}^{(M-1) / 2}, 1, \overbrace{0, \cdots, 0}^{(M-1) / 2}]$.

From $\boldsymbol{v}(k)$ and $\boldsymbol{w}(k)$, we can obtain the output of BED, as shown below:

$$
\hat{y}(k)=\boldsymbol{v}(k) \boldsymbol{w}^{T}(k)=\hat{y}_{R}(k)+j \hat{y}_{I}(k)
$$

where $(\cdot)^{T}$ represents the transpose operator, and $\hat{y}_{R}(k)$ and $\hat{y}_{I}(k)$ denote the real and imaginary parts of the BED output, respectively.

\subsection{The conventional IOWL-BED}

The structure of IOWL-BED is shown in Fig. 1. For MMA, the cost function is defined as $[43,44]$ :

$$
J_{M}(k)=J_{R}(k)+J_{I}(k)
$$

where $J_{i}(k)$ is the cost function for $\hat{y}_{i}(k), i \in\{R, I\}$ and can be given by:

$$
J_{i}(k)=E\left\{\left[\hat{y}_{i}^{2}(k)-G_{2, i}\right]^{2}\right\}
$$

where $i \in\{R, I\}, G_{2, i}$ can be calculated with $G_{2, i}=E\left\{d_{i}^{4}(k)\right\} / E\left\{d_{i}^{2}(k)\right\}, d_{R}(k)$ represents the real part of $d(k)$, and $d_{I}(k)$ is the imaginary part. For the BED, the total error is resolved by the difference between the BED output and the signal statistic of the transmitted signal and can be expressed as:

$$
e(k)=e_{R}(k)+j e_{I}(k)
$$

where

$$
\begin{aligned}
& e_{R}(k)=\hat{y}_{R}(k)\left(\hat{y}_{R}^{2}(k)-G_{2, R}\right) \\
& e_{I}(k)=\hat{y}_{I}(k)\left(\hat{y}_{I}^{2}(k)-G_{2, I}\right)
\end{aligned}
$$

According to the $J_{M}(k)$, the optimization of BED is achieved via minimizing of the mean square error $(\mathrm{MSE})$ for $e_{R}(k)$ and $e_{I}(k)$. Iterative algorithm-based stochastic gradient can

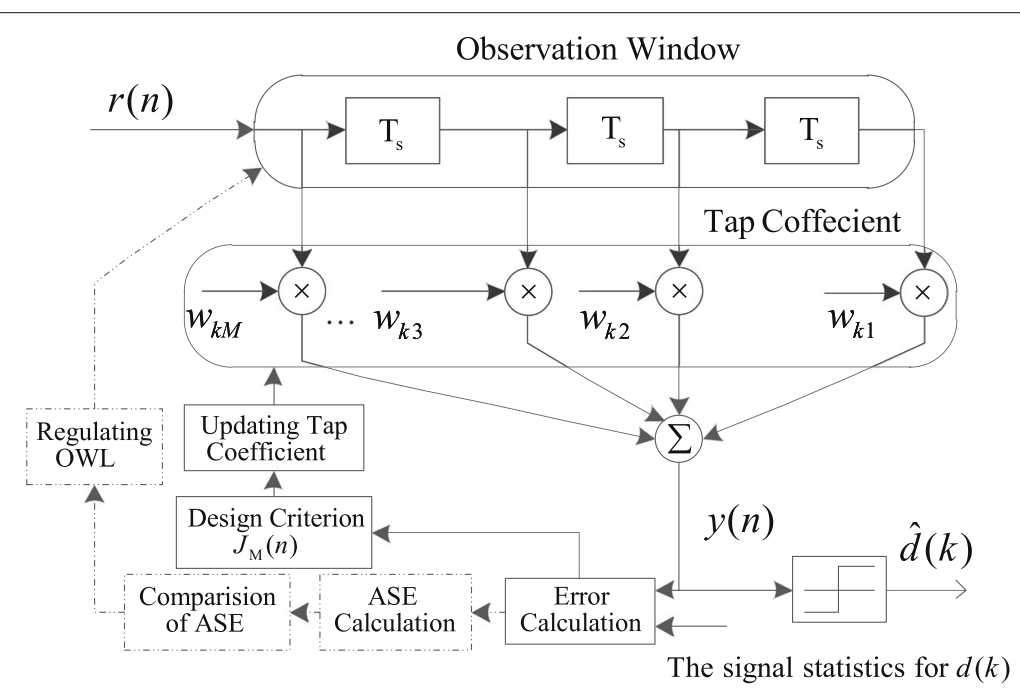

Fig. 1 The structure of VOWL-BED (the solid line denotes IOWL-BED) 
be used to simplify the problem of MSE minimization. Here, the normalized least mean square (NLMS) algorithm is employed to settle the optimization problem as below [45]:

$$
\boldsymbol{w}(k+1)=\boldsymbol{w}(k)-\frac{\lambda}{\rho+\|\boldsymbol{v}(k)\|_{2}^{2}} e(k) \boldsymbol{v}^{*}(k)
$$

where $\boldsymbol{w}(k)$ denotes the complex-valued tap coefficient vector of BED, with dimensions $M \times 1 ; \rho>0$ is a small value whose object is to preclude numerical difficulties when the input signal vector $\boldsymbol{v}(k)$ is very small; and $\lambda$ should satisfy [45]:

$$
0<\lambda<2 \frac{\operatorname{Re}\left\{E\left[\zeta(k) e^{*}(k) /\|\boldsymbol{v}(k)\|^{2}\right]\right\}}{E\left[|e(k)|^{2} /\|\boldsymbol{v}(k)\|^{2}\right]}
$$

where $\zeta(k)=\boldsymbol{\psi}^{H}(k) \boldsymbol{v}(k), \boldsymbol{\psi}(k)=\boldsymbol{w}_{o}-\boldsymbol{w}(k), \boldsymbol{w}_{o}$ denotes the optimum tap coefficient vector.

\section{Method: the proposed variable observation window length blind equalization detector}

The previous studies have focused on IOWL blind equalization detector. In this section, a VOWL-BED is devised, and the detector structure is given in Fig. 1. Compared with the existing non-blind method for real value signals in [36], (1) the devised detector can deal with complex value QAM signals, and (2) the proposed detector is based on blind equalization, rather than traditional equalization with training sequence.

Under the same conditions, when the OWL of BED is different, the output $\hat{y}(k)$ of detector is also different. At each OWL, the evident error $e(k)$ can be calculated with Eq. (8). Based on the MMSE criterion, the mean square error of error signal $e(k)$ can be used as a measure for the performance of BED at a certain OWL:

$$
\operatorname{MSE}(k)=E\left[|e(k)|^{2}\right]=\frac{\sum_{j=1}^{k} e^{2}(j)}{k}
$$

From the Eq. (13), it can be seen that a division operation is required in the end. But in fact, even if no division operation is performed, the accumulated squared error (ASE) can still measure the BED performance. Therefore, to reduce the computational complexity, the regulation of OWL can use ASE as the measure standard, and for a certain OWL, the ASE is given by:

$$
\operatorname{ASE}(k)=\sum_{j=1}^{k} e^{2}(j)
$$

In the process of OWL regulation, the input sequence is divided into equal segments. The length of each segment, which is used to calculate ASE for a certain OWL, is set as $N_{t}$. Theoretically, with the increase of the number of segments, the ASE ASE( $k$ ) obtained with each segment sequence will become smaller and smaller:

$$
\operatorname{ASE}_{N}(k)<\operatorname{ASE}_{N-1}(k)
$$

where

$$
\begin{aligned}
\operatorname{ASE}_{N}(k) & =\sum_{i=1}^{k} \gamma^{k-i}\left|y_{R, N}(k)\left(y_{R, N}^{2}(k)-G_{2, R}\right)+j y_{I, N}(k)\left(y_{I, N}^{2}(k)-G_{2, I}\right)\right|^{2} \\
\operatorname{ASE}_{N-1}(k) & =\sum_{i=1}^{k} \gamma^{k-i}\left|y_{R, N-1}(k)\left(y_{R, N-1}^{2}(k)-G_{2, R}\right)+j y_{I, N-1}(k)\left(y_{I, N-1}^{2}(k)-G_{2, I}\right)\right|^{2}
\end{aligned}
$$


where $\gamma$ is a forgetting factor used to weight the relative importance of recent and preceding outputs of BED, $\gamma \leq 1 ; y_{m, L}(l)$ denotes $l$ th output of the VOWL-BED for the $L$ th segment input sequence, $m \in\{R, I\}$; and $\operatorname{ASE}_{L}(k)$ is the ASE of the $L$ th segment input sequence.

The OWL adjustment process of VOWL-BED can be described as below:

If $\operatorname{ASE}_{N}(k) \leq \eta_{u} \mathrm{ASE}_{N-1}(k)$, the OWL increases $q$ taps:

$$
\begin{aligned}
& \boldsymbol{v}^{\prime}(k)=[u(k-1+q) \cdots, u(k-1), u(k-2), \cdots, u(k-M-q)] \\
& \boldsymbol{w}^{\prime}(k)=[\overbrace{00 \cdots 0}^{q}, \boldsymbol{w}(k), \overbrace{00 \cdots 0}^{q}] \\
& \boldsymbol{w}^{\prime}(k+1)=\boldsymbol{w}^{\prime}(k)-\frac{\lambda}{\rho+\left\|\boldsymbol{v}^{\prime}(k)\right\|_{2}^{2}} e^{\prime}(k) \boldsymbol{v}^{\prime}(k)^{*} \\
& \text { If } \operatorname{ASE}_{N}(k) \geq \eta_{d} \operatorname{ASE}_{N-1}(k), \text { the OWL decreases } q \text { taps: } \\
& \boldsymbol{v}^{\prime \prime}(k)=[u(k-1-q), u(k-2-q), \cdots, u(k-M+q)] \\
& \boldsymbol{w}^{\prime \prime}(k)=\left[w_{k(1+q)}, w_{k(2+q)}, \cdots, w_{k(M-q)}\right] \\
& \boldsymbol{w}^{\prime \prime}(k+1)=\boldsymbol{w}^{\prime \prime}(k)-\frac{\lambda}{\rho+\left\|\boldsymbol{v}^{\prime \prime}(k)\right\|_{2}^{2}} e^{\prime \prime}(k) \boldsymbol{v}^{\prime \prime}(k)^{*}
\end{aligned}
$$

where $\eta_{d}$ needs to meet $\eta_{d} \leq 1$, and $\eta_{u}$ and $\eta_{d}$ should satisfy $\eta_{u} \leq \eta_{d}$. The function of $\eta_{u}$ and $\eta_{d}$ is to determine the sum of adjustments to increase or decrease the OWL of BED according to the improvement or deterioration of ASE. The closer the values of $\eta_{u}$ and $\eta_{d}$ are, the more frequently the OWL will be adjusted by the detector.

In the adjustment process, the proposed detector continually calculates and compares the successive ASE for different OWL, then evaluates the impact that the last added length of observation window has on the SMSE level. If $\operatorname{ASE}_{N}(k)$ is much less than $\operatorname{ASE}_{N-1}(k)$, then increasing length of observation window is most likely to improve the detection performance; therefore, the OWL should be increased. If the $\mathrm{ASE}_{N}(k)$ is similar or bigger than $\operatorname{ASE}_{N-1}(k)$, the result indicates that the last increased length of observation window has little effect on the improvement of detection performance, and thus, the increased length should be removed.

In addition, it is worth noting that when the OWL of VOWL-BED and IOWLBED is the same, the incremental complexity caused by adjustment of OWL is finite. This is because for VOWL-BED, the adjustments of OWL only require multiplication, subtraction, and addition operations.

\section{Simulation results and discussion}

In this section, we present simulation results for the OWL regulation verification and the performance comparison of different approaches. The Bellhop underwater acoustic channel model is used in the simulation. In the model, the carrier frequency is $12 \mathrm{KHz}$, the range between transmitter and receiver is set as $200 \mathrm{~m}$, both transmitter and receiver are located at a depth of $10 \mathrm{~m}$, and the wave height is set as $0.2 \mathrm{~m}$. We assume that the information bit frame length is 500, and the transmitter adopts 4-QAM mode. For IOWL$B E D$, the OWL is set as 7. For the VOWL-BED, the initial length of observation window 
is set as 1 , the segment length $N_{t}$ is 10 bits, and the parameter $q$ of each OWL regulation is set as 2 .

\subsection{Verification of OWL regulation}

First of all, for a given UAC envelope, the optimum OWL is acquired by the simulation approach. The specific channel profile is generated with the Bellhop model. The influences of OWL on steady-state MSE (SMSE) are showed in Fig. 2; each point on the curve is acquired through averaging the SMSE on every data packet. The output SMSE of BED can be computed by SMSE $=E\left[|e(n)|^{2}\right]=\frac{\sum_{i=1}^{n} e^{2}(i)}{n}$ after the blind equalization algorithm achieves convergence. As shown in Fig. 2, the OWL can severely affect the SMSE performance of BED. In this paper, taking into account the implementation complexity, we define the optimum OWL according to the minimum requirement, which means that the performance approximating to the optimal SMSE can be obtained with the minimum OWL. From Fig. 2, it can be observed that when OWL is probably set between 13 and 20, the detector achieves extremely similar SMSE performance, and all the obtained performances are close to optimal SMSE. On the basis of the definition of the optimum OWL, we can obtain the optimum OWL, which is about 13.

Then, we would validate the OWL regulation capability of VOWL-BED. The evolving curve of OWL is acquired with the approach of averaging all the evolving curves, which are obtained with different data packets. Figure 3 shows the adaptive adjustment process for OWL with VOWL-BED. In the simulation, for (16) and (17), $\gamma$ is set to 0.999. In Eqs. (11), (20), and (23), $\lambda$ and $\rho$ are set to 0.25 and 0.6 , respectively. $\eta_{u}$ is set as 0.98 , and $\eta_{d}$ is set to 0.989 . In the simulation of OWL adjustment, the same underwater acoustic channel used in Fig. 2 is adopted. From Fig. 3, we can see that after VOWL-BED adaptively adjusted the OWL, the OWL can finally converge to the optimal OWL, which is

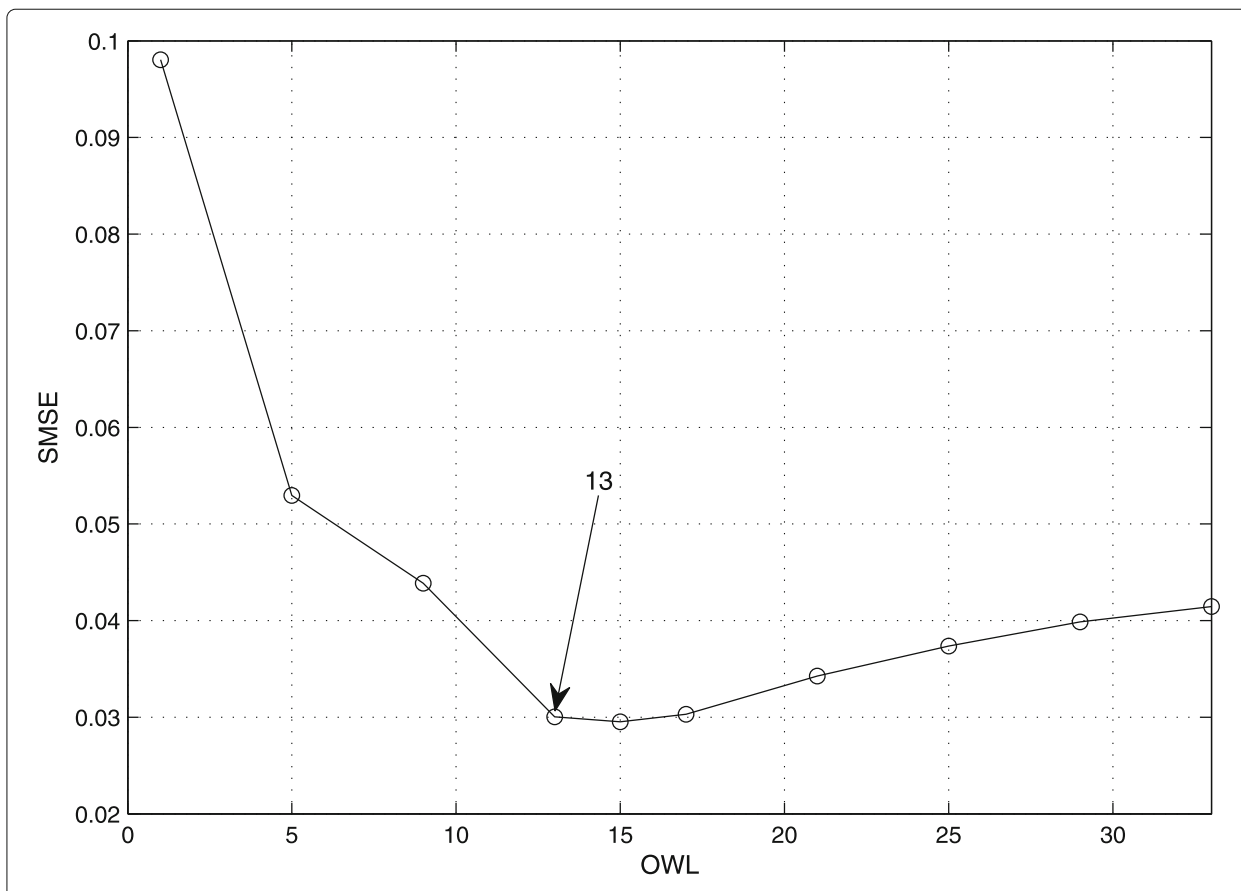

Fig. 2 SMSE performance for IOWL-BED with different OWL at SNR $=15 \mathrm{~dB}$ 


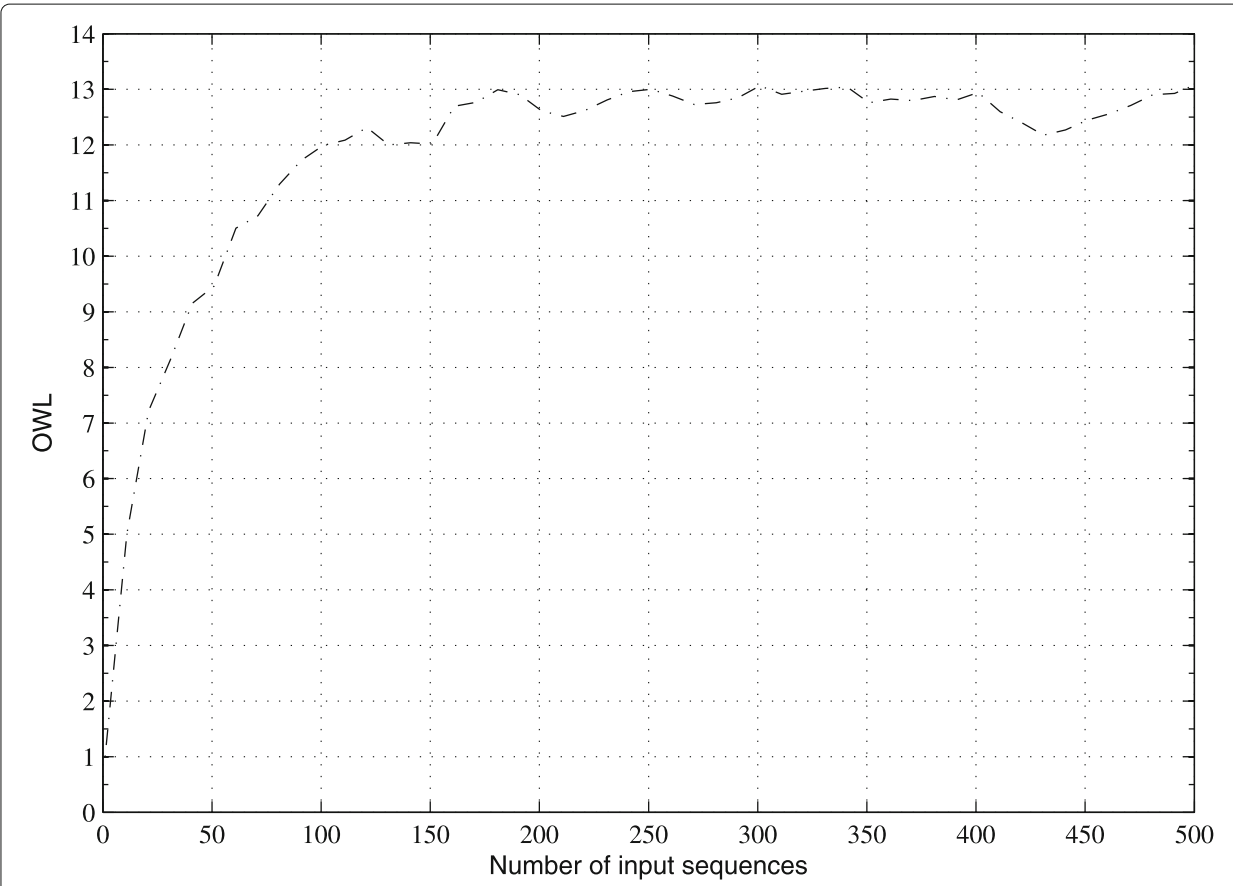

Fig. 3 OWL evolution curve for VOWL-BED at SNR $=15 \mathrm{~dB}$

consistent with the result of Fig. 2. Based on the above results, for the VOWL-BED, the capability of OWL regulation is verified.

\subsection{Comparison of BER and convergence performance}

In this section, the comparisons of BER and convergence performance of different blind equalization approaches are given. To compare the two performances, we built the Monte Carlo simulation based on the Bellhop model. In the simulation, 500 data packets are transmitted; by way of the process of averaging 500 BER and convergence curves, the BER and convergence curves are obtained.

Figure 4 depicts the BER performance of IOWL-BED and VOWL-BED schemes. It is seen that compared with IOWL-BED, the VOWL-BED achieves much better BER performance with about $10 \mathrm{~dB}$ gain around $B E R=10^{-2}$. This is because the OWL of BED plays an important role in influencing the BER performance, and the OWL can be adaptively adjusted by the proposed VOWL-BED on the basis of the given UAC envelope. Figure 5 compares the convergence curve of the proposed VOWL-BED and the conventional IOWL-BED. It can be observed from Fig. 5 that the convergence rate of VOWL-BED is very close to the IOWL-BED, but VOWL-BED can achieve smaller SMSE than IOWL$\mathrm{BED}$. The reason is that the SMSE performance of BED is severely affected by the length of observation window, and the proposed VOWL-BED can dynamically regulate and finally obtain the optimum OWL.

\section{Conclusion}

In this paper, a VOWL-BED for underwater acoustic communication is proposed. Considering the influence of UAC envelope difference on system performance, the proposed detector can dynamically regulate the OWL on the basis of the given channel envelope 


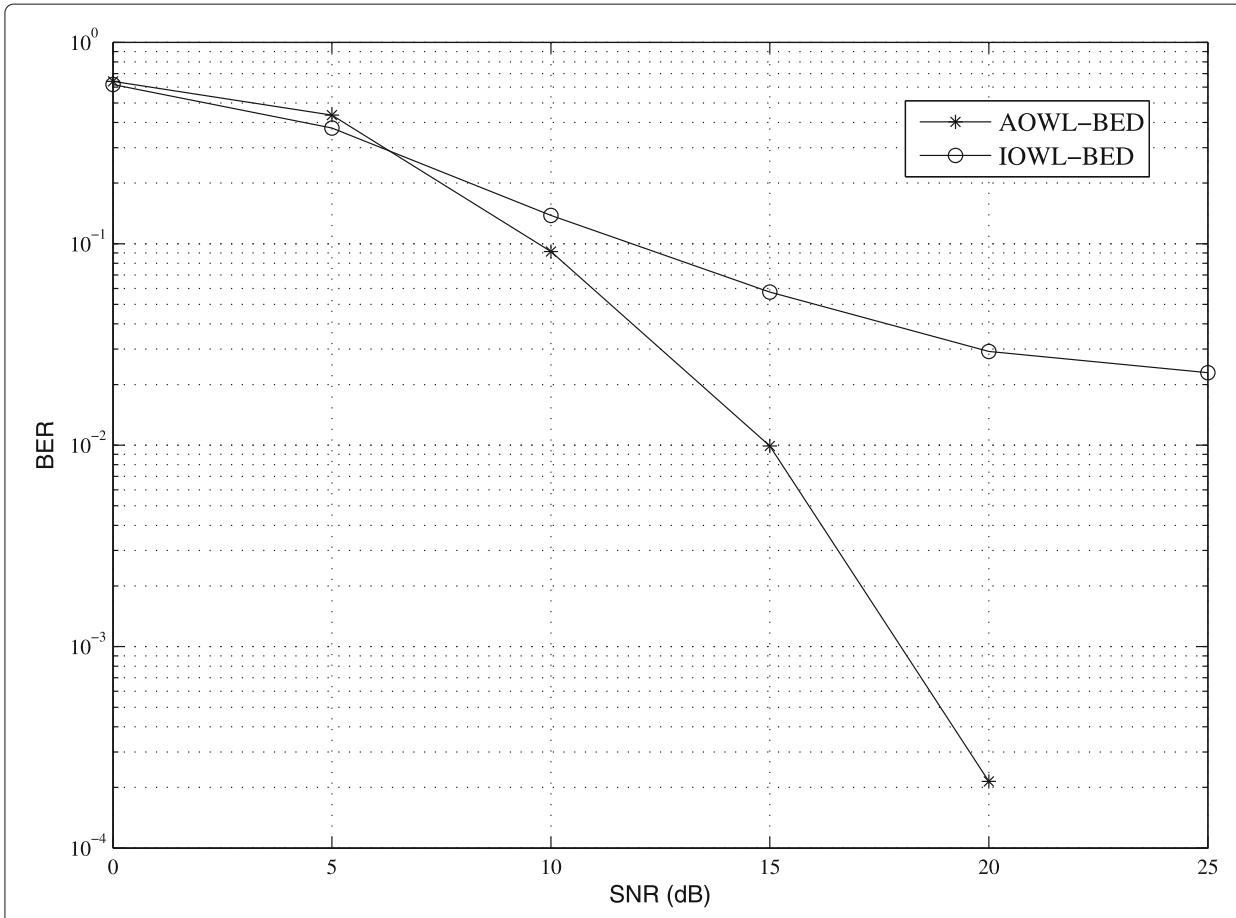

Fig. 4 BER performance comparison of VOWL-BED and IOWL-BED

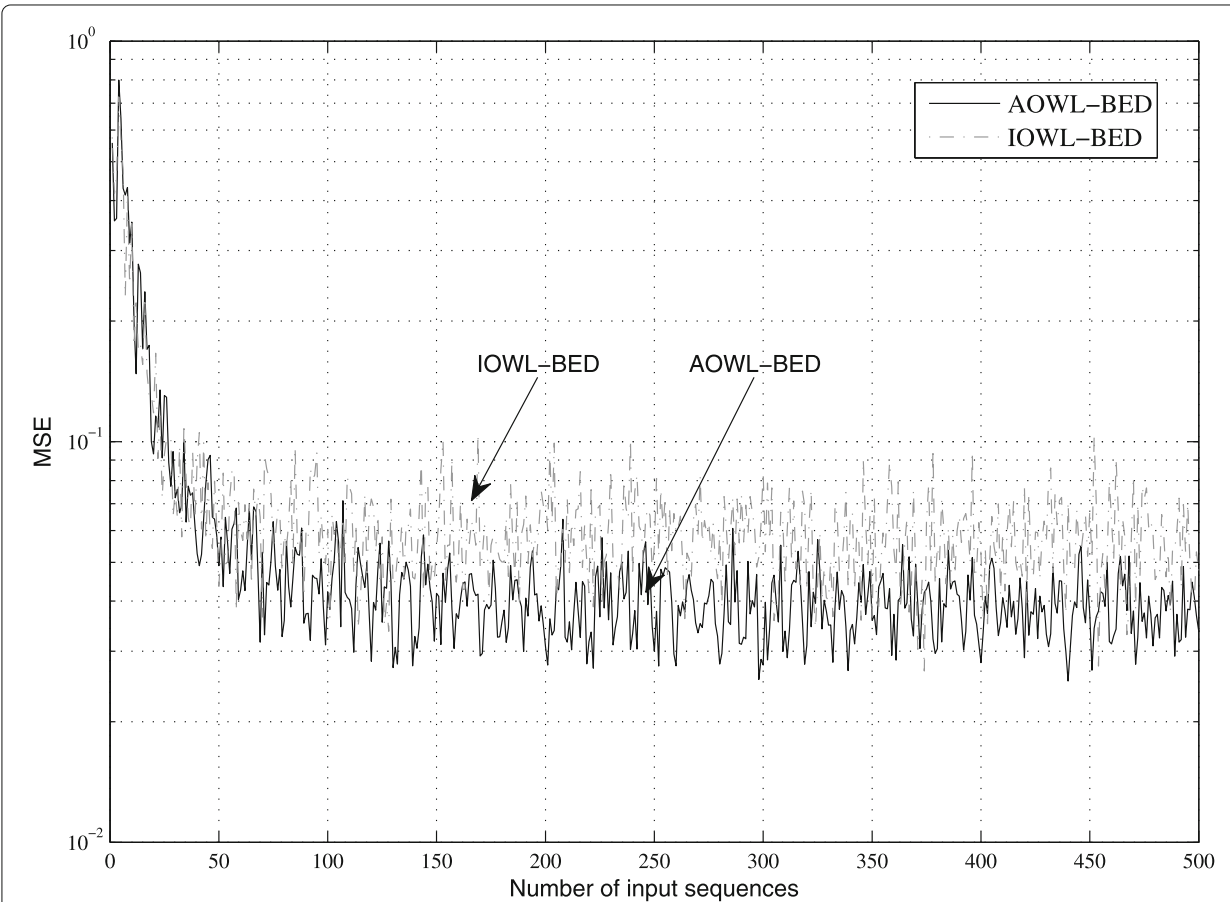

Fig. 5 Convergence performance comparison of VOWL-BED and IOWL-BED at SNR $=15 \mathrm{~dB}$ 
and finally converge to the optimum OWL. In addition, the proposed detector is realized based on blind equalization algorithm, and the training sequences are not needed. Therefore, the transmission efficiency can be greatly improved, which make the proposed detector more suitable for practical underwater acoustic communication systems. Simulation results prove the OWL regulation ability of the VOWL-BED. Simulation results likewise illustrate that the performance of VOWL-BED outperforms that of the conventional IOWL-BED.

\begin{abstract}
Abbreviations
UAC: Underwater acoustic channel; UWAC: Underwater acoustic communication; OWL: Observation window length; BED: Blind equalization detector; VOWL-BED: Variable observation window length blind equalization detector; IOWL-BED: Invariable observation window length blind equalization detector; ISI: Intersymbol interference; MMSE: Minimum mean square error; ED: Equalization detector; CMA: Constant modulus algorithm; MMA: Multimodulus algorithm; ASE: Accumulated squared error; QAM: Quadrature amplitude modulation; MSE: Mean square error; SMSE: Steady-state mean square error
\end{abstract}

\title{
Acknowledgements
}

The authors would like to thank the anonymous referees for their helpful suggestions.

\section{Authors' contributions}

ZL conceived and designed the study. FB and ZT performed the experiments. ZL wrote the paper. All authors read and approved the manuscript.

\section{Authors' information}

Zhiyong Liu was born in Henan, China, in 1979. He received the Ph.D. degree in information and communication engineering from Harbin Institute of Technology at Shenzhen, Shenzhen, China, in 2010. From 2010 to 2016, he was a Lecturer at the School of Information Science and Engineering, Harbin Institute of Technology at Weihai, where he is currently an Associate Professor. His research interests include signal processing for wireless communications, cooperative communication, and underwater acoustic communication.

Fan Bai received the B.E. degree in information and communication engineering from Hebei University, Baoding, China, in 2017. She is currently studying in the School of Information and Electrical Engineering at Harbin Institute of Technology (Weihai) for a M.E. degree. Her research interests include underwater acoustic communication, rateless coding, and blind equalization.

Zhoumei Tan received the B.E. degree in information and communication engineering from Jilin University, Changchun, China, in 2018. She is currently studying in the School of Information and Electrical Engineering at Harbin Institute of Technology (Weihai) for a M.E. degree. Her research interests include cooperative communication, underwater acoustic communication, and multiuser detection.

\section{Funding}

The work was supported by National Natural Science Foundation of China (61871148), Research and Innovation Foundation of Weihai (2019KYCXJJYB04) and Shandong Provincial Natural Science Foundation (ZR2016FM02).

\section{Availability of data and materials}

We decided that the data does not need to be shared since all data have been obtained through the simulation using the MATLAB program.

\section{Competing interests}

The authors declare that they have no competing interests.

Received: 14 January 2019 Accepted: 21 June 2020

Published online: 06 July 2020

\section{References}

1. S. Aliesawi, C. Tsimenidis, B. Sharif, M. Johnston, Iterative multiuser detection for underwater acoustic channels. IEEE J. Ocean. Eng. 36(4), 728-744 (2011)

2. G. Zhang, H. Dong, Spatial diversity in multichannel processing for underwater acoustic communications. Ocean Eng. 38(14-15), 1611-1623 (2011)

3. Z. Zhou, Z. Peng, P. Xie, J. H. Cui, Z. Jiang, Exploring random access and handshaking techniques in underwater wireless acoustic networks. EURASIP J. Wirel. Commun. Netw. 2013(1), 95 (2013)

4. J. G. Proakis, Adaptive equalization techniques for acoustic telemetry channels. IEEE J. Ocean. Eng. 16(1), 21-31 (1991)

5. M. Stojanovic, J. Catipovic, J. G. Proakis, Adaptive multichannel combining and equalization for underwater acoustic communications. J. Acoust. Soc. Am. 94(3), 1621-1631 (1993)

6. T. C. Yang, Correlation-based decision-feedback equalizer for underwater acoustic communications. IEEE J. Ocean. Eng. 30(4), 865-880 (2005)

7. F. Chen, S. Lin, B. Zheng, Q. Li, M. Wen, Y. Liu, F. Ji, Minimum symbol-error rate based adaptive decision feedback equalizer in underwater acoustic channels. IEEE Access. 5, 25147-25157 (2017) 
8. J. Labat, O. Macchi, C. Laot, Adaptive decision feedback equalization: can you skip the training period? IEEE Trans. Commun. 46(7), 921-930 (1998)

9. A. G. Bessios, Compound compensation strategies for wireless data communications over the multimodal acoustic ocean waveguide. IEEE J. Ocean. Eng. 21(2), 167-180 (1996)

10. K. C. Blom, H. S. Dol, A. B. Kokkeler, G. J. Smit, in 2016 IEEE Third Underwater Communications and Networking Conference (UComms), Blind equalization of underwater acoustic channels using implicit higher-order statistics (IEEE, 2016), pp. 1-5

11. J. Labat, J. Trubuil, M. Nicot, in IEEE Oceanic Engineering Society. OCEANS'98. Conference Proceedings, Blind decision feedback equalization application to underwater communication systems, vol. 2 (IEEE, 1998), pp. 805-809

12. J. Trubuil, G. Lapierre, T. Le Gall, J. Labat, in OCEANS'02 MTS/IEEE, Real-time high data rate acoustic link based on spatio-temporal blind equalization: the TRIDENT acoustic system, vol. 4 (IEEE, 2002), pp. 2438-2443

13. J. Xi, S. Yan, L. Xu, J. Tian, in 2015 IEEE International Conference on Signal Processing, Communications and Computing (ICSPCC), Bidirectional blind equalization based on the constant modulus and subspace-based algorithms (IEEE, 2015), pp. 1-5

14. H. S. Hung, C. C. Chang, in Oceans' 04 MTS/IEEE Techno-Ocean'04, Blind adaptive equalizer for underwater communications, vol. 1 (IEEE, 2004), pp. 34-39

15. Y. Wang, Z. Zhang, J. Liu, in Proceedings of OCEANS 2005 MTS/IEEE, Experimental study on variable structure adaptive equalizer for underwater communication system (IEEE, 2005), pp. 652-656

16. J. Gomes, V. Barroso, in OCEANS 2000 MTS/IEEE Conference and Exhibition. Conference Proceedings, Acoustic channel equalization results for the ASIMOV high-speed coherent data link (IEEE, vol. 2, 2000), pp. 1437-1442

17. R. Weber, A. Waldhorst, F. Schulz, J. F. Bohme, in MTS/IEEE Oceans 2001. An Ocean Odyssey. Conference Proceedings, Blind receivers for MSK signals transmitted through shallow water (IEEE, vol. 4, 2001), pp. 2183-2190

18. A. G. Bessios, F. M. Caimi, in Proceedings of OCEANS'94, Multipath compensation for underwater acoustic communication (IEEE, vol. 1, 1994), pp. 317-322

19. L. Cazzanti, D. Egnor, G. S. Edelson, A. Das, in OCEANS 2010 MTS/IEEE SEATTLE, Improved multipath robustness of DFH modulation in the underwater acoustic channel (IEEE, 2010), pp. 1-6

20. Y. Guo, J. Ji, in 2010 Second Pacific-Asia Conference on Circuits, Communications and System, Segmentation error function based variable momentum factor blind equalization algorithm (IEEE, vol. 1, 2010), pp. 121-124

21. J. Wang, H. Huang, C. Zhang, J. Guan, in 2009 WRI Global Congress on Intelligent Systems, A study of the blind equalization in the underwater communication (IEEE, vol. 3, 2009), pp. 122-125

22. F. Tong, X. M. Xu, D. S. Chen, in 2006 International Conference on Wireless Communications, Networking and Mobile Computing, Blind channel equalization with order estimation based on evolutionary algorithm (IEEE, 2006), pp. 1-3

23. T. Zhu, Y. Wang, in 2008 4th International Conference on Wireless Communications, Networking and Mobile Computing, A variable step-size dual-mode blind equalization algorithm based on Cadzow Theorem (IEEE, 2008), pp. 1-3

24. J. Li, J. Lu, J. Zhao, in IEEE 10th International Conference On Signal Processing Proceedings, A robust constant modulus algorithm in alpha-stable noise environments (IEEE, 2010), pp. 1589-1592

25. W. Rao, K. M. Yuan, Guo Y.C., C. Yang, in 2008 9th International Conference on Signal Processing, A simple constant modulus algorithm for blind equalization suitable for 16-QAM signal (IEEE, 2008), pp. 1963-1966

26. X. W. Zhang, W. Rao, in 2008 Fifth International Conference on Fuzzy Systems and Knowledge Discovery, New concurrent blind equalization algorithm suitable for high-order QAM signals (IEEE, 2008), pp. 177-181

27. X. L. Ning, Z. Liu, Z. K. Liu, L. S. Zhang, in 2011 International Conference on Image Analysis and Signal Processing, New super-exponential iteration blind equalization algorithm for underwater acoustic communications (IEEE, 2011), pp. 468-473

28. W. Rao, W. Q. Tan, D. S. Li, G. X. Dai, F. Xia, L. Fan, J. B. Liu, H. J. Xu, et al, in 2009 International Conference on Wireless Communications and Signal Processing, Concurrent blind equalization suitable for 16-QAM signal (IEEE, 2009), pp. 1-5

29. F. Schulz, in OCEANS 2015-Genova, Improvement of blind multichannel receivers for underwater acoustic communications by delay-based equalizer initialization (IEEE, 2015), pp. 1-7

30. R. Weber, J. E. Bohme, in Sensor Array and Multichannel Signal Processing Workshop Proceedings, 2002, Adaptive super-exponential methods for blind multichannel equalization (IEEE, 2002), pp. 585-589

31. J. Zhu, Y. Guo, C. Yang, in IEEE 10th International Conference On Signal Processing Proceedings, Decision feedback blind equalization algorithm based on joint combining frequency diversity (IEEE, 2010), pp. 263-266

32. N. Al-Dhahir, J. Cioffi, MMSE decision-feedback equalizers: finite-length results. IEEE Trans. Inf. Theory. 41(4), 961-975 (1995)

33. N. Al-Dhahir, A. Sayed, The finite-length multi-input multi-output MMSE-DFE. IEEE Trans. Signal Process. 48(10), 2921-2936 (2000)

34. Y. Gong, C. Cowan, An LMS style variable tap-length algorithm for structure adaptation. IEEE Trans. Signal Process. 53(7), 2400-2407 (2005)

35. Y. Zhang, N. Li, J. Chamber, A. Sayed, Steady-state performance analysis of a variable tap-length LMS algorithm. IEEE Trans. Signal Process. 56(2), 839-845 (2008)

36. F. Riera-Palou, J. Noras, D. Cruickshank, Linear equalizers, with dynamic and automatic length selection. Electron. Lett. 37(25), 1553-1554 (2001)

37. Y. Gu, K. Tang, H. Cui, LMS algorithm with gradient descent filter length. IEEE Signal Process. Lett. 11(3), 305-307 (2004)

38. X. Wei, D. Cruickshank, B. Mulgrew, F. Riera-Palou, A unified approach to dynamic length algorithms for adaptive linear equalizers. IEEE Trans. Signal Process. 55(3), 908-920 (2007)

39. Z. Liu, Q. Zhang, G. Liu, Adjustable observation window length multi-branch joint detector for cooperative communication. IEEE Commun. Lett. 19(5), 859-862 (2015)

40. M. Pajovic, J. Preisig, Performance analysis and optimal design of multi-channel equalizer for underwater acoustic communications. IEEE J. Ocean. Eng. 40(4), 759-774 (2015)

41. P. Qarabaqi, M. Stojanovic, Statistical characterization and computationally efficient modeling of a class of underwater acoustic communication channels. IEEE J. Ocean. Eng. 38(4), 701-717 (2013) 
42. F. Jia, E. Cheng, F. Yuan, in International Conference on Systems and Informatics (ICSAI 2012), The study on time-variant characteristics of under water acoustic channels, (2012), pp. 1650-1654

43. J. Yuan, K. Tsai, Analysis of the multimodulus blind equalization algorithm in QAM communication systems. IEEE Trans. Commun. 53(9), 1427-1431 (2005)

44. J. Yang, J. J. Werner, G. Dumont, The multimodulus blind equalization and its generalized algorithms. IEEE J. Sel. Areas Commun. 20(6), 997-1015 (2002)

45. H. Simon, Adaptive filter theory. (Prentice Hall, 2005), pp. 324-325. 4th edition

\section{Publisher's Note}

Springer Nature remains neutral with regard to jurisdictional claims in published maps and institutional affiliations.

Submit your manuscript to a SpringerOpen ${ }^{\circ}$ journal and benefit from:

- Convenient online submission

Rigorous peer review

- Open access: articles freely available online

- High visibility within the field

- Retaining the copyright to your article

Submit your next manuscript at $\gg$ springeropen.com 